\title{
Commentary: Proteomics Analysis Revealed that Crosstalk between Helicobacter pylori and Streptococcus mitis May Enhance Bacterial Survival and Reduces Carcinogenesis
}

\author{
Paweł Krzyżek* \\ Department of Microbiology, Wrocław Medical University, Wrocław, Poland
}

Keywords: Helicobacter pylori, Streptococcus, gastric cancer, stomach, virulence, coccoid bacteria, co-culture

\section{A commentary on}

Proteomics Analysis Revealed that Crosstalk between Helicobacter pylori and Streptococcus mitis May Enhance Bacterial Survival and Reduces Carcinogenesis

OPEN ACCESS

Edited by:

Angel Angelov,

Technische Universität München,

Germany

Reviewed by:

Lee-Ann H. Allen,

University of lowa, United States

*Correspondence:

Pawel Krzyżek

krojcerpawel@gmail.com

Specialty section:

This article was submitted to

Systems Microbiology,

a section of the journal

Frontiers in Microbiology

Received: 11 October 2017 Accepted: 17 November 2017 Published: 29 November 2017

Citation:

Krzyżek P (2017) Commentary:

Proteomics Analysis Revealed that Crosstalk between Helicobacter pylori

and Streptococcus mitis May

Enhance Bacterial Survival and

Reduces Carcinogenesis.

Front. Microbiol. 8:2381.

doi: 10.3389/fmicb.2017.02381 by Khosravi, Y., Loke, M. F., Goh, K. L., and Vadivelu, J. (2016). Front. Microbiol. 7:1462. doi: 10.3389/fmicb.2016.01462

For a long time, it was thought that the stomach is a sterile organ, free from the presence of microorganisms (Nardone and Compare, 2015). A breakthrough in thinking about stomach as a site possible for colonization was the discovery of Marshall and Warren (1984), who managed to isolate Helicobacter pylori, a spiral Gram-negative bacterium, from human gastric biopsies. Since then, many researchers have focused their attention on the gastric microbiota studies. It was determined that the amount of microorganisms inhabiting this niche counts of $10^{2}-10^{4} \mathrm{CFU} / \mathrm{ml}$ and varies in different stomach regions. Firmicutes and Proteobacteria prevail within the gastric mucosa, while Firmicutes, Bacteroidetes, and Actinobacteria dominate in the gastric juice (Ianiro et al., 2015; Nardone and Compare, 2015). The presence of non- $H$. pylori microbes suggests the possibility that gastric microflora may interact with $H$. pylori and modulate physiology and morphology of this bacterium. Therefore, data from researches determining such dependencies are extremely valuable.

An example of one such relevant research was the analysis of Streptococcus mitis, a physiological inhabitant of the human gastrointestinal tract, and $H$. pylori co-culture effect on the proteomes of both bacteria (Khosravi et al., 2016). The idea to determine the impact of S. mitis on $H$. pylori physiology was derived from previous researchers' observations that $S$. mitis may induce morphological transformation of $H$. pylori from spiral to coccoid form (Khosravi et al., 2014). Coculture of both bacteria significantly changed $H$. pylori proteomic profile, including the increase of proteins involved in DNA repair and genetic rearrangements, and the reduction of proteins responsible for metabolism and antioxidant activity (Khosravi et al., 2016). I believe that at this point Authors made a large mental jump, claiming that lower levels of antioxidant enzymes in $H$. pylori may be associated with a decrease in oxidative stress in the stomach tissue and subsequently lower risk of the cancer development. The conclusion of this study, as reflected in the title, was that $S$. mitis may contribute to a reduction in the $H$. pylori-induced carcinogenesis. The hypothesis although not necessarily incorrect, should be confirmed by experiments in later parts 
of this article, or be made as a minor suggestion in the Discussion section, giving the opportunity to verify this hypothesis (by a team of these or other researchers). In my opinion, it seems that: (1) the conditions in the intracellular environment (in this case $H$. pylori) are different from those in the extracellular environment (gastric mucosa niche), and there is no simple relation between conditions within both regions, (2) the decrease of proteins associated with $H$. pylori antioxidant activity does not necessarily have an adversely effect on the production of virulence factors inducing the gastric mucosa inflammation. Rejection or confirmation of the Khosravi et al. (2016) hypothesis can be made, for example, by the co-culture of both bacteria (H. pylori and S. mitis) in the presence of eukaryotic cell lines, physiological, and with induced inflammatory or neoplastic changes. Reduction of inflammation/carcinogenesis intensity in eukaryotic cells during $H$. pylori and S. mitis co-culture, in compared to $H$. pylori monoculture control, could provide evidence of Authors finding and open a new pathway in in vitro and in vivo studies.

The genus Streptococcus belong to microflora naturally occurring in the human body, especially in the digestive tract. For this reason, these bacteria are commonly used as probiotics, i.e., microorganisms with beneficial effects on the human body functions. This includes acting as an immunomodulatory, anti-inflammatory, and anti-allergic agent and protecting against gastrointestinal pathogens infection (including H. pylori) (Markowiak and Slizewska, 2017). For example, Engen et al. (2017) have shown that S. mitis cell lysate was capable of initiating wound healing by the aryl hydrocarbon receptor activation (AhR) and AhR-dependent leukocyte recruitment, involved in the remodeling of damaged tissues.

There are also studies showing the potential of Streptococcus to initiate pathogenic processes. Significantly more frequent isolation and increased numbers of these microorganisms, including $S$. mitis, have been observed in patients with antral gastritis (Li et al., 2009), as well as with gastric (Eun et al., 2014; Choi et al., 2017; Sohn et al., 2017), esophageal (Narikiyo et al., 2004; Chen et al., 2015), and oral squamous cancers (Mager et al., 2005). The relationship between the presence of these bacteria and the progression of the above-mentioned

\section{REFERENCES}

Abdulamir, A. S., Hafidh, R. R., and Abu Bakar, F. (2011). The association of Streptococcus bovis/gallolyticus with colorectal tumors: the nature and the underlying mechanisms of its etiological role. J. Exp. Clin. Cancer Res. 30:11. doi: 10.1186/1756-9966-30-11

Chen, X., Winckler, B., Lu, M., Cheng, H., Yuan, Z., Yang, Y., et al. (2015). Oral microbiota and risk for esophageal squamous cell carcinoma in a high-risk area of China. PLoS ONE 10:e0143603. doi: 10.1371/journal.pone. 0143603

Choi, H.-I., Choi, J.-P., Seo, J., Kim, B. J., Rho, M., Han, J. K., et al. (2017). Helicobacter pylori-derived extracellular vesicles increased in the gastric juices of gastric adenocarcinoma patients and induced inflammation mainly via specific targeting of gastric epithelial cells. Exp. Mol. Med. 49:e330. doi: $10.1038 / \mathrm{emm} .2017 .47$ pathologies is not known. Both the possibility of stimulation of chemokines involved in the monocytes and neutrophils recruitment (Narikiyo et al., 2004) and the presence of very active alcohol dehydrogenase $(\mathrm{ADH})$, which contributes to the high local concentrations of carcinogenic acetaldehyde (Kurkivuori et al., 2007), are suggested. One of the well-known examples of species from the genus Streptococcus, able to directly induce neoplasms, is Streptococcus gallolyticus spp. gallolyticus. The procarcinogenic potential of this bacterium includes the capacity of selective adhesion to tumor-altered tissue (colonic mucous), induction of increased cell proliferation, and activation of cytokines (IL-1, IL-8, IFN- $\gamma$ ) and transcriptional factors (NF-кB) associated with exacerbate inflammation (Abdulamir et al., 2011; Kumar et al., 2017).

The ability of Streptococcus mitis to promote gastric carcinogenesis, if any, does not need to be directly related to the virulence factors presence and the initiation of pathological changes by these microorganisms. Participation in the oncogenesis initiation may be indirect, consisting the induction of morphological transition of $H$. pylori into a coccoid form. This model is in agreement with the observations made by Authors in another study (Loke et al., 2016). H. pylori spherical forms have been shown to have a higher level of proteins associated with the intensification of carcinogenesis than spiral forms. Hence, Streptococcus mitis could even contribute in vivo to the enhancement of $H$. pylori-mediated gastric cancer development. This model is hypothetical and should be proven experimentally.

The statement presented in this commentary does not in any way diminish the relevance or value of the research conducted by Khosravi et al. (2016). On the contrary, it simply seeks to understand the complexity of bacteria-bacteria and host-bacteria interactions, and makes a suggestion for further research in this area.

\section{AUTHOR CONTRIBUTIONS}

After having carefully read the recent Frontiers in Microbiology article by Yalda Khosravi and coworkers, upon which this manuscript is based, the Author (PK) has autonomously and independently written the present Commentary.

Engen, S. A., Rørvik, G. H., Schreurs, O., Blix, I. J., and Schenck, K. (2017). The oral commensal Streptococcus mitis activates the aryl hydrocarbon receptor in human oral epithelial cells. Int. J. Oral Sci. 9, 145-150. doi: 10.1038/ijos.2017.17

Eun, C. S., Kim, B. K., Han, D. S., Kim, S. Y., Kim, K. M., Choi, B. Y., et al. (2014). Differences in gastric mucosal microbiota profiling in patients with chronic gastritis, intestinal metaplasia, and gastric cancer using pyrosequencing methods. Helicobacter 19, 407-416. doi: 10.1111/hel.12145

Ianiro, G., Molina-Infante, J., and Gasbarrini, A. (2015). Gastric microbiota. Helicobacter 20, 68-71. doi: 10.1111/hel.12260

Khosravi, Y., Dieye, Y., Loke, M. F., Goh, K. L., and Vadivelu, J. (2014). Streptococcus mitis induces conversion of Helicobacter pylori to coccoid cells during co-culture in vitro. PLoS ONE 9:e112214. doi: 10.1371/journal.pone.0112214

Khosravi, Y., Loke, M. F., Goh, K. L., and Vadivelu, J. (2016). Proteomics analysis revealed that crosstalk between Helicobacter pylori and Streptococcus mitis may 
enhance bacterial survival and reduces carcinogenesis. Front. Microbiol. 7:1462. doi: 10.3389/fmicb.2016.01462

Kumar, R., Herold, J. L., Schady, D., Davis, J., Kopetz, S., MartinezMoczygemba, M., et al. (2017). Streptococcus gallolyticus subsp. gallolyticus promotes colorectal tumor development. PLoS Pathog. 13:e1006440. doi: 10.1371/journal.ppat.1006440

Kurkivuori, J., Salaspuro, V., Kaihovaara, P., Kari, K., Rautemaa, R., Grönroos, L., et al. (2007). Acetaldehyde production from ethanol by oral streptococci. Oral Oncol. 43, 181-186. doi: 10.1016/j.oraloncology.2006.02.005

Li, X.-X., Wong, G. L.-H., To, K.-F., Wong, V. W.-S., Lai, L. H., Chow, D. K.L., et al. (2009). Bacterial microbiota profiling in gastritis without Helicobacter pylori infection or non-steroidal anti-inflammatory drug use. PLoS ONE 4:e7985. doi: 10.1371/journal.pone.0007985

Loke, M. F., Ng, C. G., Vilashni, Y., Lim, J., and Ho, B. (2016). Understanding the dimorphic lifestyles of human gastric pathogen Helicobacter pylori using the SWATH-based proteomics approach. Sci. Rep. 6:26784. doi: 10.1038/ srep26784

Mager, D. L., Haffajee, A. D., Devlin, P. M., Norris, C. M., Posner, M. R., and Goodson, J. M. (2005). The salivary microbiota as a diagnostic indicator of oral cancer: a descriptive, non-randomized study of cancer-free and oral squamous cell carcinoma subjects. J. Transl. Med. 3:27. doi: 10.1186/14795876-3-27

Markowiak, P., and Slizewska, K. (2017). Effects of probiotics, prebiotics, and synbiotics on human health. Nutrients 9:1021. doi: 10.3390/nu90 91021
Marshall, B. J., and Warren, J. R. (1984). Unidentified curved bacilli in the stomach of patients with gastritis and peptic ulceration. Lancet 1, 1311-1315. doi: 10.1016/S0140-6736(84)91816-6

Nardone, G., and Compare, D. (2015). The human gastric microbiota: is it time to rethink the pathogenesis of stomach diseases? United Eur. Gastroenterol. J. 3, 255-260. doi: 10.1177/2050640614566846

Narikiyo, M., Tanabe, C., Yamada, Y., Igaki, H., Tachimori, Y., Kato, H., et al. (2004). Frequent and preferential infection of Treponema denticola, Streptococcus mitis, and Streptococcus anginosus in esophageal cancers. Cancer Sci. 95, 569-574. doi: 10.1111/j.1349-7006.2004.tb02488.x

Sohn, S.-H., Kim, N., Jo, H. J., Kim, J., Park, J. H., Nam, R. H., et al. (2017). Analysis of gastric body microbiota by pyrosequencing: possible role of bacteria other than Helicobacter pylori in the gastric carcinogenesis. J. Cancer Prev. 22, 115-125. doi: 10.15430/JCP.2017.22.2.115

Conflict of Interest Statement: The author declares that the research was conducted in the absence of any commercial or financial relationships that could be construed as a potential conflict of interest.

Copyright (C) 2017 Krzyżek. This is an open-access article distributed under the terms of the Creative Commons Attribution License (CC BY). The use, distribution or reproduction in other forums is permitted, provided the original author(s) or licensor are credited and that the original publication in this journal is cited, in accordance with accepted academic practice. No use, distribution or reproduction is permitted which does not comply with these terms. 